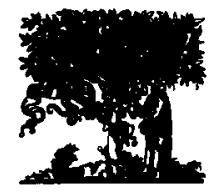

ELSEVIER

Fluid Phase Equilibria 117 (1996) 26-32

\title{
Application of the GFD EOS on VLE Calculations of Polymer Solutions
}

\author{
Chyau-Song Wu, Muoi Tang and Yan-Ping Chen
}

\author{
Department of Chemical Engineering \\ National Taiwan University \\ Taipei, Taiwan, Republic of China
}

Keywords: theory, vapor-liquid equilibria, equation of state, polymer solution

\begin{abstract}
The generalized Flory-dimer (GFD) equation of state (EOS) developed by Hall and coauthors is employed in this study on the vapor-liquid equilibrium (VLE) calculations of polymer solutions. This EOS is applied in real fluid systems where the pure component parameters of the low molecular weight compounds and polymers are regressed using experimental data. Simple mixing rules are employed in this study for binary fluid mixtures of polymers and solvents. It is observed that the GFD EOS yields good VLE calculation results on polymer solutions. The calculated results of the activities and solubilities of solvents in polymer solutions are very satisfactory. The absolute average deviations from the GFD EOS are smaller than those from the SAFT EOS and the Sanchez- Lacombe EOS. The GFD EOS is also compared with other EOS or activity coefficient models on their calculated results of the activity coefficients of solvents at infinite dilution. The GFD EOS again shows better agreement with experimental data than that from other models.
\end{abstract}

\section{Introduction}

Vapor-liquid equilibria (VLE) on polymer solutions are calculated by using either the equations of state (EOS) or the activity coefficient models. Recently, several EOS have been proposed for phase equilibrium calculations of polymer systems from various theoretical considerations (e.g., the SAFT EOS (Huang and Radosz, 1990); the GFD EOS (Yethiraj and Hall, 1991; Bokis et al., 1994)). The GFD EOS has been applied to real fluid systems and satisfactory pure component calculation results have been reported (Wu and Chen, 1994). In this research, the GFD EOS is extended to real fluid mixtures of solvents and polymers. VLE calculation results of polymer solutions from the GFD EOS are presented in this work. The comparisons of this EOS with other EOS or activity coefficient models are also investigated in this study.

\section{Method of calculations}

The GFD EOS expresses the compressibility factor of a m-mer fluid by two reference components of a monomer and a dimer (Yethiraj and Hall, 1991):

$Z_{m}=Z_{1}+\frac{V_{e, m}-V_{e, 1}}{V_{e, 2}-V_{e, 1}}\left(Z_{2}-Z_{1}\right)$ 
where $\mathrm{V}$ is the excluded volume and its value for a monomeric hard sphere $\left(\mathrm{V}_{01}\right)$, a dimer or a trimer molecule $\left(\mathrm{V}_{\mathrm{e2}}\right.$ or $\left.\mathrm{V}_{\mathrm{e} 3}\right)$ has been shown by Hall and co-workers. The compressibility factor $\mathrm{Z}$ in Eq. (1) is composed of two parts due to the repulsive and attractive contributions between hard spheres. The repulsive hard sphere monomer and dimer EOS (Carnahan and Starling, 1969; Tildesley and Streett, 1980), and the attractive EOS for tangent spheres via square-well interaction forces (Yethiraj and Hall, 1991) were employed in the expression of the GFD EOS. There are three pure fluid parameters in the GFD EOS: the number of segment, $\mathrm{m}$; the molar volume parameter of a hard sphere, $v^{\circ}$; and the energy parameter, $\varepsilon / \mathrm{kT}$. Wu and Chen (1994) have regressed the pure fluid parameters of the GFD EOS for low molecular weight compounds and polymers. It was found that the GFD EOS can very satisfactorily calculate the pure fluid properties. Wu and Chen (1994) expressed the volume and energy parameters of the GFD EOS in a temperature-dependent form:

$v^{\circ}=v^{00}+0.005 T$

$\varepsilon / k T=\varepsilon^{\circ} / k T-0.04 T$

The GFD EOS parameters, $\mathrm{m}, \mathrm{v}^{\infty}$, and $\varepsilon^{\circ} / \mathrm{kT}$, for more than one hundred pure low molecular weight compounds and polymers were reported by Wu and Chen (1994). In this work, we extend our pure fluid calculations to polymer solutions. In this VLE calculations, the equal fugacity criterion is satisfied:

$f_{1}^{v}=\hat{f}_{1}^{L}$

where subscript 1 denotes the solvent in a polymer solution. In the VLE calculations of polymer solutions, the polymer molecule is assumed to be nonvolatile and only pure solvent exists in the vapor phase. The following mixing rules, which are usually employed in mixture computations, are also used in this work:

$m=\Sigma x_{i} m_{i}$

$m v^{o}=\Sigma x_{i} m_{i} v_{i}^{o}$

$\varepsilon / k T=\Sigma \Sigma s_{i} s_{j}\left(\varepsilon_{i j} / k T\right)$

where the segment fraction $\mathbf{s}$ and the pseudopair energy parameter $\varepsilon_{\mathrm{ij}}$ are defined as:

$s_{i}=x_{i} m_{i} /\left(\Sigma x_{k} m_{k}\right)$

$\varepsilon_{i j}=\left(\varepsilon_{i} \varepsilon_{j}\right)^{0.5}\left(1-k_{i j}\right)$

A binary parameter $k_{i j}$ is included in the mixing rules. The optimal $k_{\mathfrak{j}}$ parameters are obtained by minimizing the following objective function:

$O . F .=\Sigma \mid\left(x^{c a l}-x^{\exp )} / x^{\exp } \mid\right.$

In the polymer solution systems, $x$ in Eq. (10) denotes the activity or the solubilities of the solvent molecule. The summation in Eq. (10) is over all the experimental data points.

Results and discussion

The GFD EOS is applied in this work on VLE calculations of polymer solutions. Many pure fluid parameters of the GFD EOS have been shown by Wu and Chen (1994). Some new pure component parameters 
of the GFD EOS are presented in Table 1 of this work. It is shown that the pure fluid saturated properties are well-correlated by the GFD EOS and the absolute average deviations in saturated pressures or liquid molar volumes are very small.

Table 1 New parameters of the GFD EOS for pure fluids regressed in this work

\begin{tabular}{|c|c|c|c|c|c|c|c|c|c|}
\hline $\begin{array}{l}\text { Pure } \\
\text { fluid }\end{array}$ & $\begin{array}{l}\text { No. of } \\
\text { data }\end{array}$ & $\begin{array}{c}\text { Temp. } \\
\mathbf{K}\end{array}$ & $\begin{array}{r}\text { Press. } \\
\text { MPa }\end{array}$ & $\mathbf{m}$ & $\begin{array}{c}\mathrm{v}^{\infty 0} \\
\mathrm{~cm}^{3} / \mathrm{mol}\end{array}$ & $\begin{array}{l}\varepsilon^{\circ} / \mathbf{k} \\
(\mathrm{K})\end{array}$ & $\begin{array}{c}\text { AADP } \\
(\%)\end{array}$ & $\begin{array}{c}\text { AADV } \\
(\%)\end{array}$ & $\begin{array}{l}\text { Data } \\
\text { ref. }\end{array}$ \\
\hline $\begin{array}{l}\text { propyl } \\
\text { acetate }\end{array}$ & 15 & $312-374$ & $\begin{array}{c}0.009- \\
0.099\end{array}$ & 2.437 & 18.471 & 342.53 & 0.15 & n.a. & 4 \\
\hline $\begin{array}{l}\text { butyl } \\
\text { acetate }\end{array}$ & 15 & $333-399$ & $\begin{array}{c}0.009- \\
0.101\end{array}$ & 2.544 & 20.875 & 355.41 & 0.04 & n.a. & 4 \\
\hline $\begin{array}{l}\text { dipropyl } \\
\text { ether }\end{array}$ & 34 & $300-361$ & $\begin{array}{c}0.009- \\
483\end{array}$ & 2.233 & 24.513 & 337.98 & 0.87 & 0.09 & 2,3 \\
\hline $\begin{array}{l}\text { diisopropyl } \\
\text { ketone }\end{array}$ & 23 & $293-372$ & $\begin{array}{c}0.001- \\
0.046\end{array}$ & 2.262 & 25.309 & 370.17 & 0.22 & 0.27 & 1 \\
\hline 3-pentanone & 11 & $285-347$ & $\begin{array}{c}0.002- \\
0.04\end{array}$ & 2.23 & 18.615 & 359 & 0.21 & 0.08 & 1 \\
\hline $\begin{array}{c}\text { nature } \\
\text { rubber(NR) }\end{array}$ & 17 & $273-298$ & $0.1-49$ & $1,586.7$ & 32.875 & 524.98 & 0.02 & n.a. & 5 \\
\hline
\end{tabular}

AADP or AADV is the absolute average deviation in vapor pressure or saturated liquid volume. Data references: (1) B. D. Smith and R. Srivastava, Thermodynamic data for pure compounds. Elsevier, Amsterdam, 1986 (2) J. Phys. Chem., 72:1057,1968 (3) T. Boublik, et al., The vapor pressures of pure substances. Elsevier, Amsterdam,1973 (4) R. C. Reid, et al., The properties of gases and liquids. McGraw-Hill, NY,1987 (5) J. Res. Nat. Bur. Stand., 68A:259,1964

Table 2 shows the calculated activities of solvents in polymer solutions. Four methods are tested including three EOS approaches (the SL(Sanchez and Lacombe, 1978), SAFT(Huang and Radosz, 1990) and GFD EOS) and a group contribution activity coefficient model (OP method, Oishi and Prausnitz, 1969). It is noticed that the GFD EOS presents satisfactory results on the VLE of polymer solutions and yields better results on more systems than those from other methods. It is also observed from Table 2 that the GFD EOS has smaller grand average or peak deviations than other methods. Fig. 1 shows the graphical comparison of the calculated activities of methyl ethyl ketone in nature rubber by various methods. The GFD EOS shows very good agreement with experimental data. Fig. 2 gives another example on the calculated activities of diethyl ketone in polypropylene. It is demonstrated in Fig. 2 that although different models give good results at low weight fractions of the solvent, the GFD EOS yields satisfactory results up to higher concentrations.

Table 3 shows the calculated infinite dilution activity coefficients of solvents in several polymer solutions. We compare the calculated results from the GFD EOS with those from the SL and SAFT EOS, and with those from two group contribution methods (UNIFAC-FV, Oishi and Prausnitz, 1978; GC-Flory, Chen et al., 1990). The binary parameters of the EOS methods shown in Table 2 are also used in the infinite dilution activity coefficient calculations and there is no empirical parameter for the two group contribution methods. It is again demonstrated that the GFD EOS gives better results for most systems while the SAFT EOS and the UNIFAC-FV method have relatively larger errors. This study also calculates the solubilities of solvents in polymers by different EOS methods. In these calculations, we found that the binary parameters of the SAFT, SL and GFD EOS can be correlated as a linear function of temperature. The linear correlations of the binary parameters are presented in Table 4. The calculated solubilities of solvents in polymers are listed in Table 5 using the generalized binary parameters. It is shown in Table 5 that all EOS yield comparable accuracy in calculating the solvent solubilities while the GFD EOS has a slightly smaller peak deviation. 


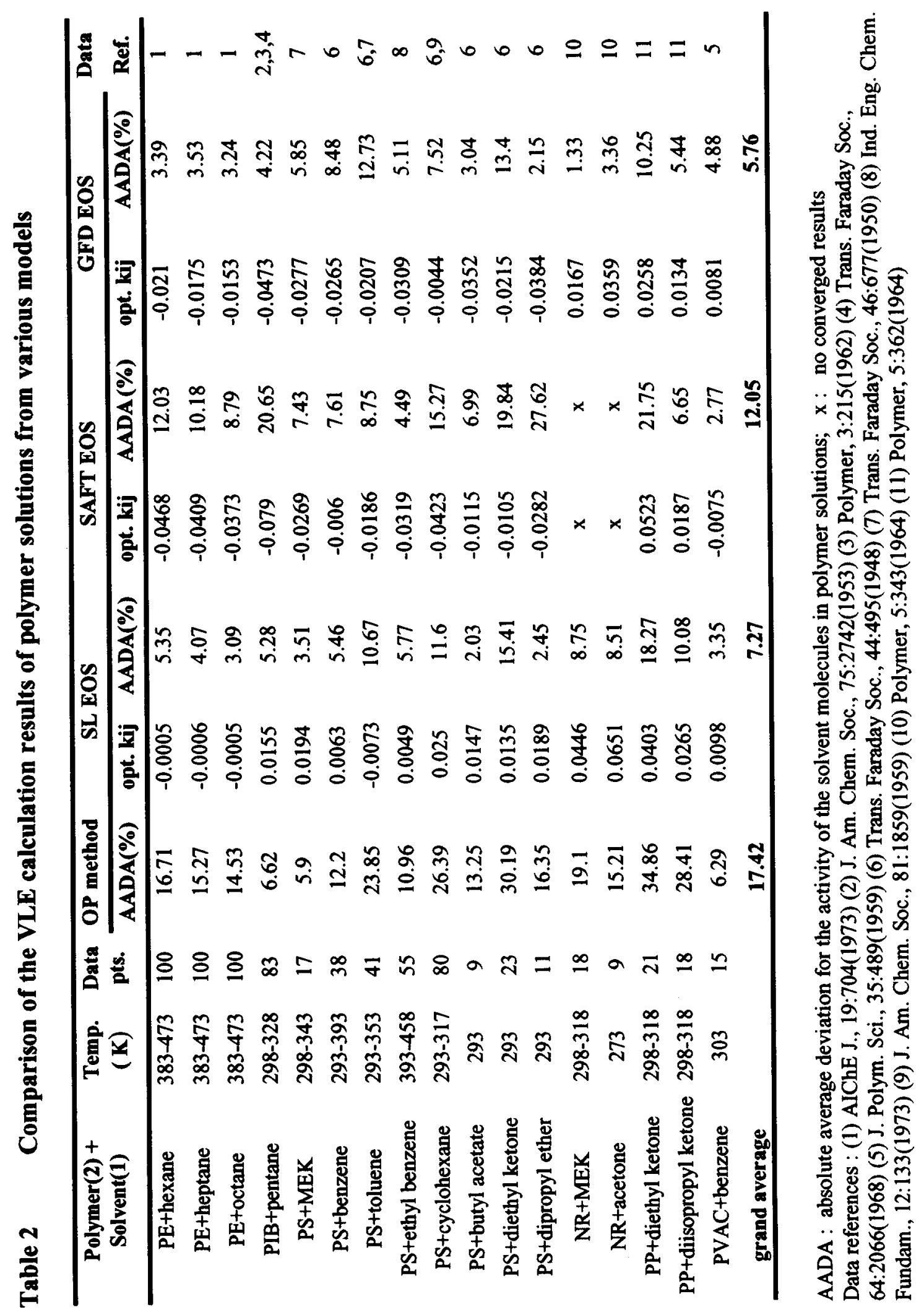




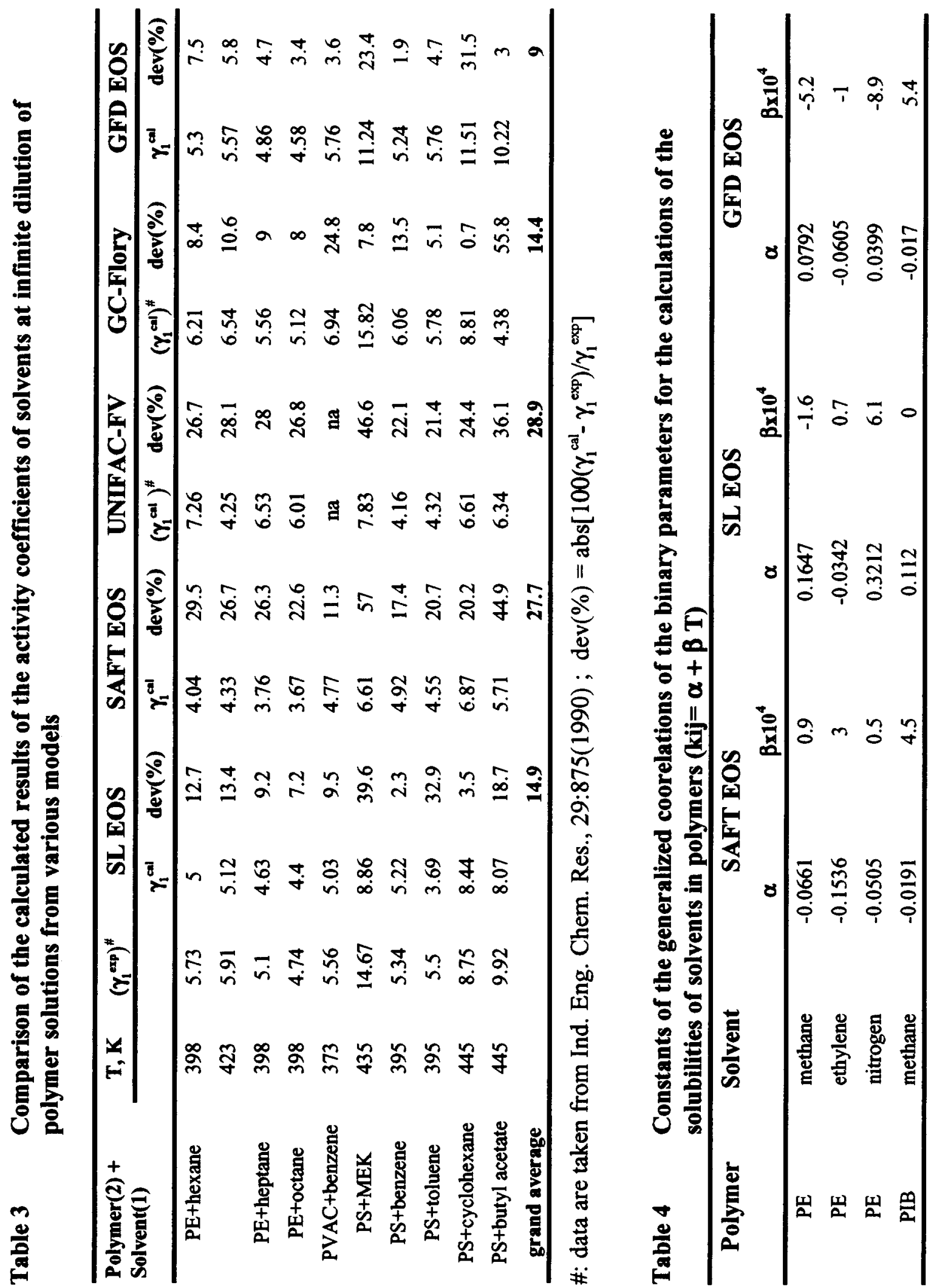




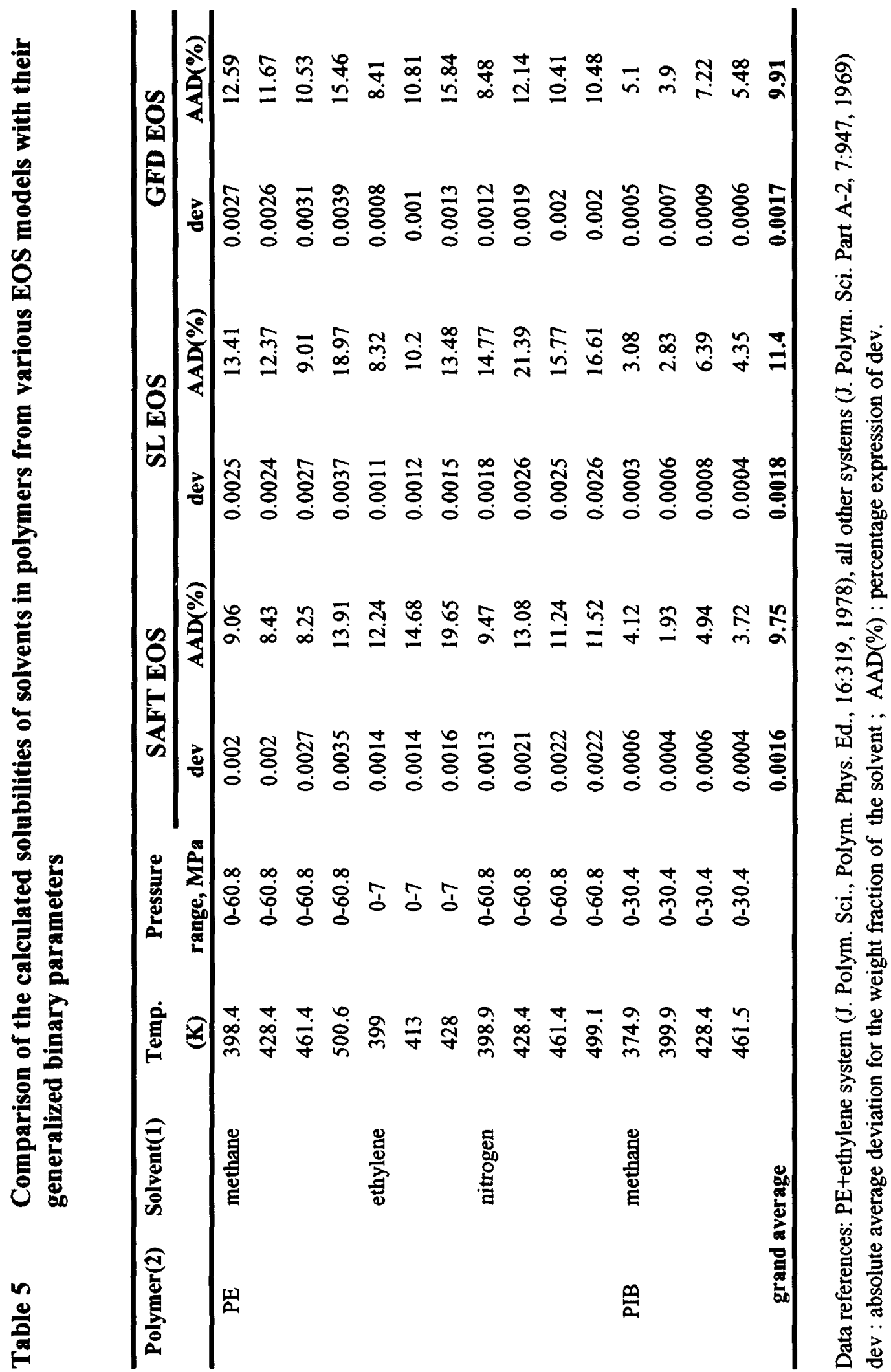




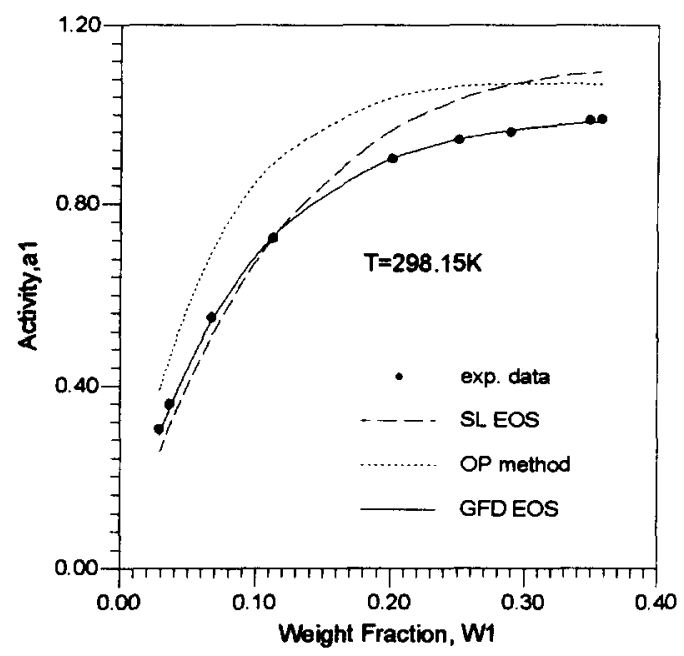

Fig 1 Comparison of the calculated activities of methyl ethyl ketone(1) in nature rubber(2)

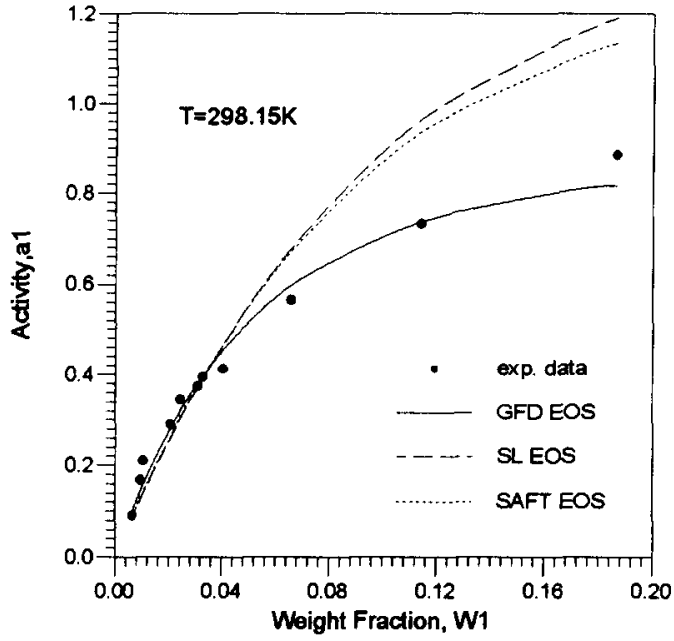

Fig. 2 Comparison of the calculated activities of diethyl ketone(1) in polypropylene(2)

\section{Conclusion}

The GFD EOS is applied in this study for VLE calculations of polymer solutions. New EOS parameters for some pure fluids are reported. The calculated results of the activities, solubilities, and the infinite dilution activity coefficients of the solvents in polymer solutions are presented. The GFD EOS gives satisfactory results and shows good agreement with the experimental data. The GFD EOS yields overall better results than those from other EOS or group contribution methods. It is shown that the GFD EOS is appropriate for the VLE calculations of polymer solutions with good accuracy.

\section{Acknowledgement}

The authors are grateful to the National Science Council, Republic of China, for supporting this research.

\section{References}

Bokis, C. P., Donohue, M. D. and Hall, K. C., 1994. Ind. Eng. Chem. Res., 33:1290-1298

Carnahan, N. F. and Starling, K. E., 1969. J. Chem. Phys., 51:635-636

Chen, F., Fredenslund, Aa. and Rasmussen, P., 1990. Ind. Eng. Chem. Res., 29:875-882

Huang, S. H. and Radosz, M., 1990. Ind. Eng. Chem. Res., 29:2284-2294

Oishi, T. and Prausnitz, J. M., 1978. Ind. Eng. Chem. Process Des. Dev., 17:333-339

Sanchez, I. C. and Lacombe, R. H., 1978. Macromolecules, 11:1145-1156

Tildesley, D. J. and Streett, W. B., 1980. Mol. Phys., 41:85-94

Wu, C. S. and Chen, Y. P., 1994. Fluid Phase Equilibria, 101:3-26

Yethiraj, A. and Hall, K. C., 1991. J. Chem. Phys., 95:8494-8506 